\title{
ARDS Outcomes in Non-Research Subjects Assessed by Generalized Prospective Trial Eligibility Criteria and Adherence to Lung-Protective Ventilation
}

\author{
Richard H Kallet, Michael S Lipnick, and Romain Pirracchio
}

\begin{abstract}
BACKGROUND: ARDS mortality is lower among subjects participating in randomized controlled trials (RCTs) compared to subjects in observational studies. Excluding potential subjects with inordinately high mortality risk is necessary to prevent masking the impact of potentially effective treatments. We inquired whether observed mortality differed between RCT-eligible and RCT-ineligible subjects managed with varying degrees of lungprotective ventilation in a non-research setting. METHODS: This single-center, retrospective, observational study utilized quality assurance data for monitoring lung-protective ventilation practices based upon National Institutes of Health ARDS Network (ARDSNet) protocols. Between 2002 and 2017, 1,975 subjects meeting the 1994 consensus criteria for acute lung injury/ARDS (later reclassified by the Berlin definition) were prospectively identified and classified as RCT-eligible or RCT-ineligible on the basis of the original ARDSNet exclusion criteria for comorbidities or moribund condition. Demographic and physiologic data from the day of ARDS onset and outcome data were studied. Survival was modeled with a mixed-effect Cox proportional hazard model adjusted for age, both illness and lung injury severity plateau pressure, and formal use of the ARDSNet ventilator protocol. The primary outcome of interest was all-cause mortality during the first $90 \mathrm{~d}$ following onset of ARDS. RESULTS: Day 90 mortality was $27.6 \%$ in RCT-eligible subjects versus $\mathbf{5 0 . 4 \%}$ in RCT-ineligible subjects (hazard ratio 0.47 [95\% CI 0.41-0.54], $P<.001$ ). Regardless of eligibility or ineligibility, achieving a plateau pressure $\leq 30 \mathrm{~cm} \mathrm{H} \mathrm{H}_{2} \mathrm{O}$ was associated with lower mortality. Overall, mortality risk was lower in subjects managed by protocol versus clinician-directed lung-protective ventilation (hazard ratio 0.60 [95\% CI 0.52-0.69], $P<.001$ ), even among those in whom plateau pressure was $\leq 30 \mathrm{~cm} \mathrm{H} \mathrm{H}_{2} \mathrm{O}$ (hazard ratio 0.64 [95\% CI $\left.0.54-0.76\right], P<.001$ ). CONCLUSIONS: Mortality in non-research, RCT-eligible subjects was substantially lower compared to RCT-ineligible subjects. Managing non-research patients with ARDS by keeping plateau pressure $\leq 30 \mathrm{~cm} \mathbf{H}_{2} \mathrm{O}$ and formal use of a lung-protective ventilation protocol significantly reduces mortality risk. Key words: acute respiratory distress syndrome; ARDSNet; lung-protective ventilation; randomized controlled trial. [Respir Care 2021;66(9):1380-1388. (C) 2021 Daedalus Enterprises]
\end{abstract}

\section{Introduction}

Since publication of the seminal trial on low tidal volume $\left(\mathrm{V}_{\mathrm{T}}\right)$ ventilation by the National Institutes of Health ARDS Clinical Trials Network (ARDSNet), ${ }^{1}$ studies have reported higher mortality in the general ARDS population managed with lung-protective ventilation compared to those in randomized controlled trials (RCT). ${ }^{2,3}$ This was largely attributed to exclusion criteria used in the latter to prevent masking the effects of potential useful treatments due to subjects with exceptionally high mortality risk. In addition, rigorous adherence to treatment protocols in RCTs are speculated to enhance mortality reduction. ${ }^{3,4}$ Furthermore, delayed recognition of ARDS in observational studies along with the small fraction of screened subjects enrolled into RCTs are cited as additional factors that limit generalizing beneficial RCT results to clinical practice. ${ }^{3}$ 
We previously reported that adopting the ARDSNet lungprotective ventilation protocol for clinical management of ARDS significantly reduced mortality in patients meeting

See the Related Editorial on Page 1498

RCT-eligibility as well as in patients meeting RCT-ineligibility criteria compared to traditional mechanical ventilation practices. ${ }^{5}$ The current study reexamines in more detail, and with a larger sample, how mortality and other patient-centered outcomes are influenced by RCT-eligibility and ineligibility criteria. ${ }^{1}$

\section{Methods}

\section{Population}

Consecutive subjects treated at San Francisco General Hospital for acute lung injury or ARDS on the basis of the American-European Consensus Conference crite$\mathrm{ria}^{6}$ (and subsequently reclassified according to the Berlin definition ${ }^{7}$ ) were entered into a quality assurance database used to monitor adoption of the ARDSNet ARMA ventilator protocol. ${ }^{1}$ Beginning in 2005 the PEEP $/ \mathrm{F}_{\mathrm{IO}_{2}}$ grid from the ARDSNet ALVEOLI trial protocol was incorporated as an option for $91 \%$ of our subjects managed by protocol. ${ }^{8}$ Continuous mandatory ventilation was the primary ventilator mode used when implementing these protocols. Protocolized management was at the ICU attending's discretion and, over a 16-y period, protocol usage averaged $74 \%$, ranging annually between $61 \%$ and $85 \%$. Patients undergoing clinician-directed lung-protective ventilation typically received a $\mathrm{V}_{\mathrm{T}}$ of $7-8 \mathrm{~mL} / \mathrm{kg}$. By policy, $\mathrm{V}_{\mathrm{T}}$ was set according to predicted body weight and corrected for compressible volume loss in the circuit.

The authors are affiliated with the Department of Anesthesia and Perioperative Care, University of California, San Francisco at Zuckerberg San Francisco General Hospital and Trauma Center, San Francisco, CA.

A version of this paper was presented by Mr Kallet at AARC Congress 2018, held December 4-7, 2018, in Las Vegas, Nevada.

Supplementary material related to this paper is available at http://www. rcjournal.com.

Mr Kallet has disclosed a relationship with Nihon Kohden. Dr Pirracchio has disclosed a relationship with General Electric HealthCare. Dr Lipnick has disclosed a relationship the US Agency for International Development.

Correspondence: Richard H Kallet MSc RRT FAARC, 2070 Fell St \#1, San Francisco, CA. 94117. E-mail: richkallet@gmail.com.

DOI: $10.4187 /$ respcare.09116

\section{QUICK LOOK}

\section{Current knowledge}

Mortality reported in the general ARDS population managed with lung-protective ventilation is higher than that reported in randomized controlled trials (RCTs) because of the need to exclude patients with comorbid conditions and excessive mortality risk. This limits the ability to generalize these findings to the ARDS population at large.

\section{What this paper contributes to our knowledge}

In a non-research setting, early identification of patients with ARDS and use of the National Institutes of Health ARDSNet ventilator protocol produced mortality rates in those who would meet RCT eligibility criteria that were similar to mortality rates reported in several ARDSNet trials. These RCT-eligible subjects had a $>50 \%$ reduction in mortality risk compared to RCT-ineligible subjects.

\section{RCT Eligibility}

One investigator (RHK) who was site clinical coordinator for the ARDSNet clinical trials group (1996-2007) screened and entered each subject into the database according to the primary source of lung injury, as well as sepsis as a co-diagnosis. Subjects were classified as either meeting or not meeting RCT-eligibility criteria as defined in the ARDSNet ARMA trial. ${ }^{1}$ Ineligibility criteria used for quality-assurance purposes were restricted to comorbid conditions likely to increase mortality, duration of mechanical ventilation, or ICU length of stay (LOS) (see the supplementary materials at http://www. rcjournal.com). No patients had been co-enrolled into any ongoing ARDSNet clinical trials between 2002 and 2008.

\section{Measurements}

The quality assurance database consisted primarily of information gathered from the day of ARDS onset including mechanical ventilation and gas exchange data, initial illness severity scores, use of ancillary ARDS therapies, as well as other demographic and outcome data. In the subset of 1,230 subjects managed with the ARDSNet protocol, additional ventilator data were collected $\sim 24 \mathrm{~h}$ after protocol initiation to assess protocol adherence.

Acute Physiology and Chronic Health Evaluation score (APACHE II), ${ }^{9}$ Simplified Acute Physiology score (SAPS II),${ }^{10}$ and lung injury score ${ }^{11}$ were calculated on the day of 
ARDS onset. Ventilator systems status checks with contemporaneous arterial blood gas data were collected within $4 \mathrm{~h}$ after ARDS onset. Measurements included respiratory system compliance $\left(\mathrm{C}_{\mathrm{RS}}\right)$ calculated as $\mathrm{V}_{\mathrm{T}}$ divided by the difference between end-inspiratory plateau pressure and PEEP $\left(\mathrm{P}_{\text {plat }}-\mathrm{PEEP}\right)$, which also was recorded as elastic driving pressure. ${ }^{12}$

Oxygenation was assessed both as the ratio of $\mathrm{P}_{\mathrm{aO}}$ to $\mathrm{F}_{\mathrm{IO}_{2}}\left(\mathrm{P}_{\mathrm{aO}_{2}} / \mathrm{F}_{\mathrm{IO}_{2}}\right)$ and as the oxygenation index, calculated as the product of mean airway pressure and the percent of inspired oxygen divided by $\mathrm{P}_{\mathrm{aO}_{2}} \cdot{ }^{13}$ Ventilation efficiency was assessed using the ventilatory ratio, calculated as $\frac{\dot{\mathrm{V}}_{\mathrm{E}} \times \mathrm{P}_{\mathrm{aCO}} \mathrm{CO}_{2}}{\text { normalized } \dot{\mathrm{V}}_{\mathrm{E}} \times 37.5}$, where $\dot{\mathrm{V}}_{\mathrm{E}}$ is minute ventilation. ${ }^{14}$

Temporal measurements included days from ICU admission to initiation of invasive mechanical ventilation, and from its initiation to ARDS onset. Duration of mechanical ventilation, ICU LOS, and hospital LOS from ARDS onset were calculated for survivors only. Approval to use our quality assurance data was granted by the University of California, San Francisco institutional review board (\#268589).

\section{Statistical Analysis}

Statistical analysis was done using PRISM 8.2.3 (Graphpad Software, La Jolla, California) and R package 3.2-10 (Available at: https://CRAN.R-project.org/ package $=$ survival). Continuous variables were expressed as either mean $\pm \mathrm{SD}$ or median and interquartile range (IQR) and were compared using either unpaired $t$ test or the Mann-Whitney test. Paired comparisons were made using either paired $t$ test or Wilcoxon sign-rank test. Categorical variables were compared using the chi square test with Yates correction. The Kruskall-Wallis test was used to compare $>2$ groups.

The primary outcome of interest was all-cause mortality during the first $90 \mathrm{~d}$ following ARDS onset. The primary comparison was between RCT-eligible subjects and RCT-ineligible subjects. Additional prospectively planned comparisons included $\mathrm{P}_{\text {plat }}$ below or above $30 \mathrm{~cm} \mathrm{H}_{2} \mathrm{O}$, Berlin classes, protocol-based versus clinician-based ventilator management, and use of ancillary therapies to support gas exchange.

Actuarial survival was displayed using Kaplan-Meier plots and compared using the log rank test. Survival was modeled using a mixed-effect Cox proportional hazard model adjusted for age, APACHE II score, Berlin class, lung injury score, $\mathrm{P}_{\text {plat }}$, ARDS etiology, concomitant sepsis, type of ICU (medical, neurologic, or surgical-trauma), number of ancillary therapies employed as fixed effect, and the year of hospitalization as random intercept. Secondary outcomes focused on the duration of mechanical ventilation and ICU and hospital LOS in survivors from the onset of ARDS. Alpha was set at 0.05 .

\section{Results}

\section{Population Characteristics}

This study analyzed data from 1,975 consecutive subjects between July 2002 and December 2017. This sample comprised 1,136 (58\%) RCT-eligible subjects and 839 (42\%) RCT-ineligible subjects. The most frequent reasons for RCT ineligibility were acute brain injury $(n=292$; $34.8 \%)$, end-stage liver disease (ie, Child's Class C) $(n=$ $153 ; 18.2 \%$ ), and perceived moribund condition at the time of initial assessment (ie, apparent refractory shock not based upon pre-hoc formal criteria) $(n=124$; $14.8 \%$ ), of whom 35 also had end-stage liver disease. Significantly more RCT-eligible subjects received care in either the medical or surgical-trauma ICU setting, whereas significantly more RCT-ineligible subjects were managed in the neurocritical care setting and also were older (Table 1). Neither gender nor racial-ethnic background were different between groups.

Although RCT-eligible subjects had significantly lower APACHE II and SAPS II scores, ARDS severity at onset was not different between eligibility groups by Berlin category, lung injury score, or those with a lung injury score $>$ 3 (ie, eligibility criteria for extracorporeal membrane oxygenation). ${ }^{15}$ RCT-eligible subjects had a higher incidence of pancreatitis, nonpulmonary sepsis, and sepsis as a co-diagnosis (Table 1).

\section{Respiratory Mechanics and Quality of Lung- Protective Ventilation}

RCT-eligible subjects had significantly higher $\mathrm{P}_{\mathrm{aO}_{2}} / \mathrm{F}_{\mathrm{IO}_{2}}$ and lower oxygenation index, weight-adjusted $\mathrm{V}_{\mathrm{T}}$, and $\mathrm{C}_{\mathrm{RS}}$ compared to RCT-ineligible subjects. However, PEEP, $\mathrm{F}_{\mathrm{IO}_{2}}$, mean airway pressure, $\mathrm{P}_{\text {plat }}$, and ventilatory ratio did not differ (Table 2). Use of the ARDSNet ventilator protocol was significantly higher in the RCT-eligible group than in the RCT-ineligible group (73\% vs $58.2 \% ; P<.001)$. ARDSNet ventilator protocol was initiated either on the day of ARDS onset or on the following day in $92.8 \%$ of RCT-eligible subjects and $90.6 \%$ of RCT-ineligible subjects $(P=.20)$.

There was a small, significant difference in PEEP among RCT eligible subjects managed with the ARDSNet protocol between the study period prior to the ALVEOLI study and the study period after the ALVEOLI study (ie, 2002-2004 vs 2005-2017): 8 (IQR 5-10) vs 10 (IQR 5$10)$, respectively $(P<.001)$. A similar trend also was observed in RCT-ineligible subjects managed with the protocol: 8 (IQR 5-10) vs 10 (IQR 5-12), respectively $(P=.06)$. 
Table 1. Subject Characteristics on Day of ARDS Onset and Outcomes

\begin{tabular}{|c|c|c|c|}
\hline & RCT-Eligible $(n=1,136)$ & RCT-Ineligible $(n=839)$ & $P$ \\
\hline \multicolumn{4}{|l|}{ Setting } \\
\hline Medical ICU & $714(62.9)$ & $438(52.2)$ & $<.001$ \\
\hline Neurologic critical care unit & $13(1.1)$ & $211(25.1)$ & $<.001$ \\
\hline Surgical ICU & $409(36)$ & $190(22.6)$ & $<.001$ \\
\hline Age, y & $50.6 \pm 16.6$ & $52.4 \pm 17.0$ & .02 \\
\hline \multicolumn{4}{|l|}{ Gender } \\
\hline Male & $849(74.7)$ & $612(72.9)$ & .97 \\
\hline Female & $310(27.3)$ & $227(27.1)$ & .92 \\
\hline \multicolumn{4}{|l|}{ Race/ethnicity } \\
\hline Black & $227(20)$ & $170(20.3)$ & .74 \\
\hline Asian/Pacific & $190(16.7)$ & $143(17)$ & .85 \\
\hline European & $460(4.5)$ & $331(39.4)$ & .78 \\
\hline Hispanic & 245 (21.6) & $180(21.4)$ & .82 \\
\hline Middle Eastern & $11(1)$ & $12(1.4)$ & .49 \\
\hline Native American & $2(0.2)$ & $4(0.5)$ & .22 \\
\hline \multicolumn{4}{|l|}{ ARDS severity (Berlin definition) } \\
\hline Mild & $181(15.9)$ & $111(13.2)$ & .11 \\
\hline Moderate & $572(50.4)$ & 419 (49.9) & .94 \\
\hline Severe & $383(33.7)$ & $309(36.8)$ & .17 \\
\hline Lung injury score & $2.5(2.3-3.0)$ & $2.5(2.3-3.0)$ & .91 \\
\hline Lung injury score $>3.0^{*}$ & $237(20.9)$ & $170(20.3)$ & .79 \\
\hline \multicolumn{4}{|l|}{ Primary ARDS etiology } \\
\hline Aspiration & $165(14.5)$ & $148(17.6)$ & .056 \\
\hline Pancreatitis & $49(4.3)$ & $7(.8)$ & $<.001$ \\
\hline Pneumonia & $381(33.5)$ & $256(3.5)$ & .26 \\
\hline Nonpulmonary sepsis & $252(22.2)$ & $142(17)$ & .008 \\
\hline Trauma & $220(19.4)$ & $174(20.7)$ & .58 \\
\hline Other & $69(6.1)$ & $112(13.3)$ & $<.001$ \\
\hline Sepsis as co-diagnosis ${ }^{\dagger}$ & $223(19.6)$ & $131(15.6)$ & .03 \\
\hline APACHE II score & $21(15-27)$ & $25(19-31)$ & $<.001$ \\
\hline SAPS II score & $45(34-59)$ & $51(40-64)$ & $<.001$ \\
\hline Time from ICU admit to initiation of mechanical ventilation, $d$ & $0(0-0)$ & $0(0-0)$ & .049 \\
\hline Time from initiation of mechanical ventilation to ARDS onset, $d$ & $0(0-1)$ & $0(0-2)$ & $<.001$ \\
\hline Time from ARDS onset to protocol initiation, $d$ & $0(0-0)$ & $0(0-0)$ & .064 \\
\hline Duration of mechanical ventilation with ARDS (survivors), d & $9(4-18)$ & $11(6-21)$ & $<.001$ \\
\hline ICU LOS with ARDS (survivors), d & $12(6-22)$ & $15(8-25)$ & $<.001$ \\
\hline Time from ARDS onset to hospital discharge (survivors), $d$ & $23(13-44)$ & $29(15-47)$ & .02 \\
\hline Mortality at day 90 & $314(27.6)$ & $423(50.4)$ & $<.001$ \\
\hline $\begin{array}{l}\text { Data are presented as } n(\%) \text {, mean } \pm \text { SD, or median (interquartile range). } \\
\text { * Extracorporeal membrane oxygenation enrollment criteria. } \\
{ }^{\dagger} \text { Incidence in those with primary ARDS etiologies of aspiration, pancreatitis, pneumon } \\
\text { APACHE = Acute Physiology and Chronic Health Evaluation score } \\
\text { SAPS = Simplified Acute Physiology Score } \\
\text { LOS = length of stay }\end{array}$ & and other. & & \\
\hline
\end{tabular}

The quality of lung-protective ventilation was not different between RCT-eligible and RCT-ineligible groups in terms of achieving $\mathrm{P}_{\text {plat }}$ and $\mathrm{V}_{\mathrm{T}}$ targets (see the supplementary materials at http://www.rcjournal.com). In addition, there was no difference between groups at ARDS onset in incidents when $\mathrm{V}_{\mathrm{T}}, \mathrm{P}_{\text {plat }}$, or elastic driving pressure reached levels believed to substantially increase ventilator-induced lung injury risk (ie, $\mathrm{V}_{\mathrm{T}} \geq 12 \mathrm{~mL} / \mathrm{kg}, \mathrm{P}_{\text {plat }} \geq 35 \mathrm{~cm} \mathrm{H}_{2} \mathrm{O}$, and elastic driving pressure $\left.>20 \mathrm{~cm} \mathrm{H}_{2} \mathrm{O}\right){ }^{1,12,16,17}$ In both study cohorts, subjects managed by protocol experienced further significant reductions in $\mathrm{V}_{\mathrm{T}}$, elastic driving pressure, and $\mathrm{F}_{\mathrm{IO}_{2}}$ as well as increased PEEP over the first $24 \mathrm{~h}$ following protocol initiation (see the supplementary materials at http://www.rcjournal.com). There was no difference in the frequency or number of ancillary therapies used to support gas exchange between RCT-eligible subjects and RCT-ineligible subjects (see the supplementary materials at http://www.rcjournal.com). 
Table 2. Mechanical Ventilation and Gas Exchange Variables on Day of ARDS Onset

\begin{tabular}{|c|c|c|c|}
\hline & RCT-Eligible $(n=1,136)$ & RCT-Ineligible $(n=839)$ & $P$ \\
\hline ARDSNet Protocol Use & $829(73)$ & $489(58.2)$ & $<.001$ \\
\hline \multicolumn{4}{|l|}{ Protocol use in: } \\
\hline Medical ICU & $516(72.3)$ & $260(59.4)$ & $<.001$ \\
\hline Neurologic critical care unit & $7(53.8)$ & $102(48.3)$ & .92 \\
\hline Surgical ICU & $306(74.8)$ & $127(66.8)$ & .053 \\
\hline Tidal volume, $\mathrm{mL}$ & $455(398-520)$ & $460(400-533)$ & .21 \\
\hline Tidal volume, $\mathrm{mL} / \mathrm{kg}$ predicted body weight & $7.1(6.1-8.1)$ & $7.4(6.2-8.2)$ & .003 \\
\hline Plateau pressure, $\mathrm{cm} \mathrm{H}_{2} \mathrm{O}$ & $24(21-28)$ & $24(21-28)$ & .78 \\
\hline PEEP, $\mathrm{cm} \mathrm{H}_{2} \mathrm{O}$ & $8(5-10)$ & $8(5-10)$ & .25 \\
\hline Driving pressure, $\mathrm{cm} \mathrm{H}_{2} \mathrm{O}^{*}$ & $16(13-19)$ & $15(12-19)$ & .047 \\
\hline$\overline{\mathrm{P}}_{\mathrm{aw}}, \mathrm{cm} \mathrm{H}_{2} \mathrm{O}$ & $15(12-18)$ & $15(12-18)$ & .63 \\
\hline $\mathrm{C}_{\mathrm{RS}}, \mathrm{mL} / \mathrm{cm} \mathrm{H}_{2} \mathrm{O}$ & $29(24-36)$ & $31(24-38)$ & .01 \\
\hline Minute ventilation, $\mathrm{L} / \mathrm{min}$ & $1.1(8.1-12.4)$ & $1.0(8.1-12.2)$ & .58 \\
\hline $\mathrm{P}_{\mathrm{aCO}_{2}}, \mathrm{~mm} \mathrm{Hg}$ & $40(35-46)$ & $40(35-45)$ & .95 \\
\hline Ventilatory ratio & $1.7(1.4-2.1)$ & $1.6(1.4-2.1)$ & .72 \\
\hline $\mathrm{pH}$ & $7.35(7.25-7.41)$ & $7.35(7.27-7.42)$ & .47 \\
\hline Base excess, $\mathrm{mEq} / \mathrm{dL}$ & $-3.8(-8.3$ to 0.4$)$ & $-3.3(-8.5$ to 1.2$)$ & .15 \\
\hline $\mathrm{F}_{\mathrm{IO}_{2}}$ & $0.80(0.60-1.0)$ & $0.80(0.60-1.0)$ & .17 \\
\hline $\mathrm{P}_{\mathrm{aO}_{2}}, \mathrm{~mm} \mathrm{Hg}$ & $87(71-114)$ & $84(70-107)$ & .02 \\
\hline $\mathrm{P}_{\mathrm{aO}_{2}} / \mathrm{F}_{\mathrm{IO}_{2}}, \mathrm{~mm} \mathrm{Hg}$ & $125(90-180)$ & $120(87-162)$ & .001 \\
\hline Oxygenation index & $11.6(7.2-17.5)$ & $12(7.7-19.5)$ & .01 \\
\hline $\begin{array}{l}\text { Data are presented as } n(\%) \text { or median (interquartile range). } \\
\text { *Difference between plateau pressure and PEEP. } \\
\overline{\mathrm{P}}_{\text {aw }}=\text { mean airway pressure } \\
\mathrm{C}_{\mathrm{RS}}=\text { respiratory system compliance }\end{array}$ & & & \\
\hline
\end{tabular}

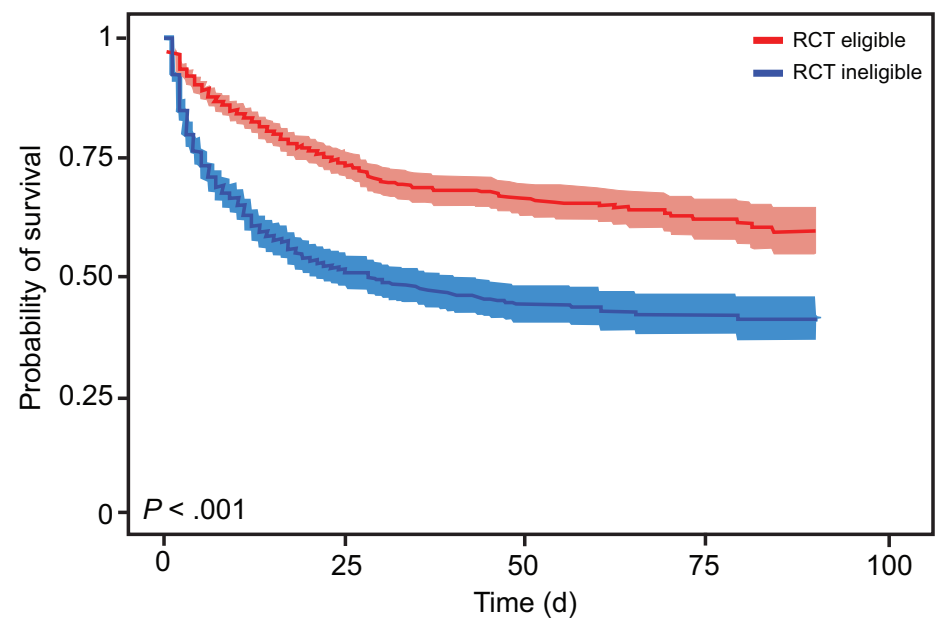

Fig. 1. Kaplan-Meier plots of the probability of $90-d$ survival with $95 \% \mathrm{Cl}$ between patients meeting randomized controlled trial (RCT) eligibility versus ineligibility criteria.

\section{Primary Outcome}

The predicted mortality based on the APACHE II scores was $40 \%$ and $55 \%$ for RCT-eligible subjects and RCTineligible subjects, respectively. Observed mortality was markedly lower in RCT-eligible subjects compared to
RCT-ineligible subjects at day 90: $27.6 \%$ versus $50.4 \%$ (hazard ratio 0.47 [95\% CI 0.41-0.54], $P<.001$ ) (Fig. 1). This was the case across both Berlin classifications and ARDS etiology, with one exception: those with pneumonia (see the supplementary materials at http://www.rcjournal. com). Among the $15 \%$ of RCT-ineligible subjects 


\section{ARDS Outcomes In Non-Research SubJects}

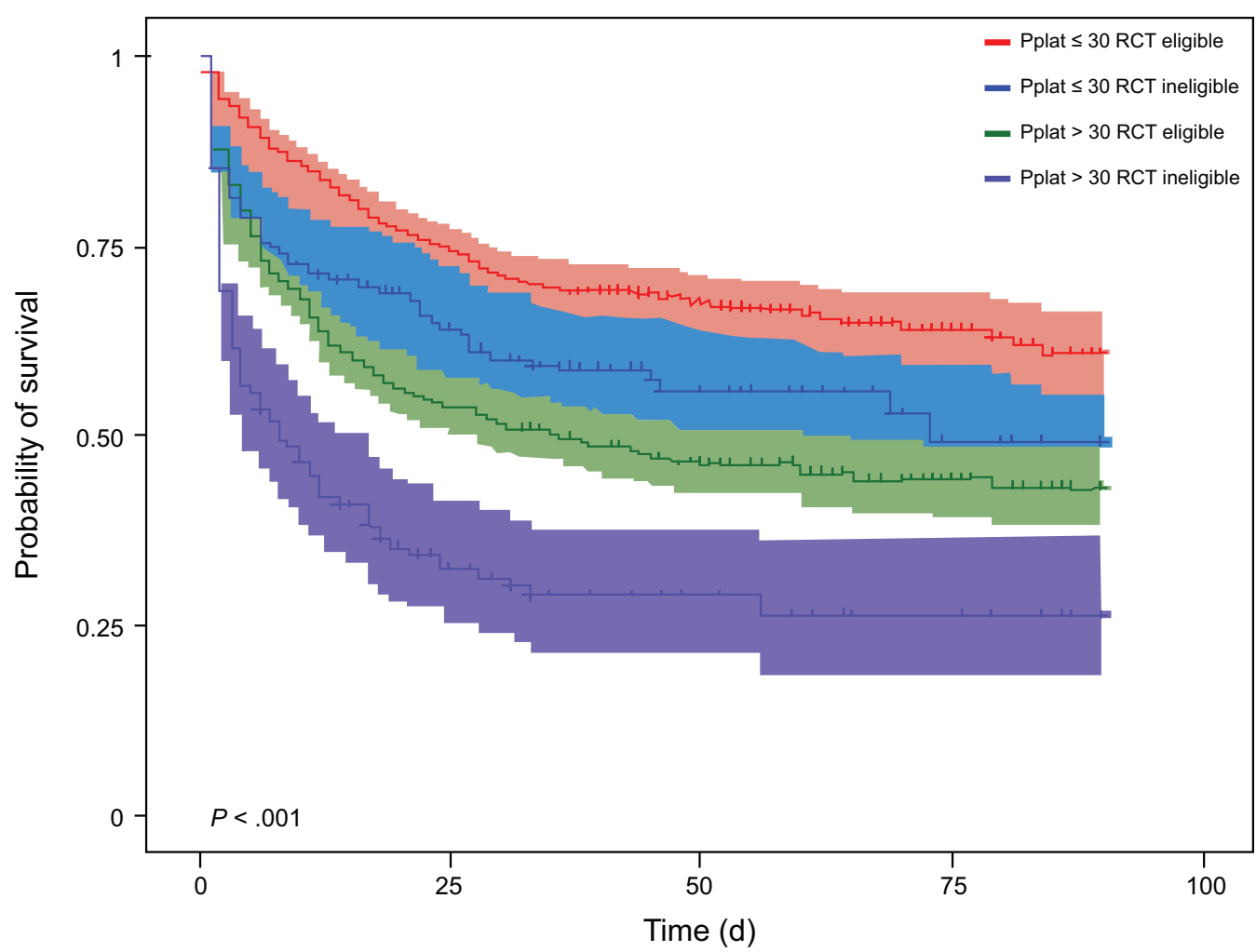

Fig. 2. Kaplan-Meier plots of the probability of $90-\mathrm{d}$ survival with $95 \% \mathrm{Cl}$ between subjects meeting randomized controlled trial (RCT) eligibility versus ineligibility criteria and a plateau pressure $\left(\mathrm{P}_{\text {plat }}\right)$ cutoff of $30 \mathrm{~cm} \mathrm{H}_{2} \mathrm{O}$.

Table 3. Adjusted Cox Proportional Hazard Model for 90-d Mortality

\begin{tabular}{lcc}
\hline \hline \multicolumn{1}{c}{ Variable } & Hazard Ratio (95\% CI) & $P$ \\
\hline Age & $1.01(1.01-1.02)$ & $<.001$ \\
APACHE II & $1.07(1.06-1.08)$ & $<.001$ \\
RCT ineligible & $1.96(1.67-2.27)$ & $<.001$ \\
Severe ARDS (Berlin definition) & $1.26(1.10-1.46)$ & .001 \\
Lung injury score & $1.00(0.83-1.19)$ & .97 \\
Plateau pressure & $0.67(0.55-0.81)$ & $<.001$ \\
ARDS etiology & $1.02(0.97-1.07)$ & .46 \\
Sepsis & $0.85(0.70-1.02)$ & .09 \\
Medical ICU & $0.73(0.64-0.83)$ & $<.001$ \\
Number of ancillary therapies & $1.11(1.03-1.21)$ & .008
\end{tabular}

APACHE $=$ Acute Physiology and Chronic Health Evaluation Score $\mathrm{RCT}=$ randomized controlled trial

considered moribund at ARDS onset, the mortality was $83 \%$, which was significantly greater than other RCT-ineligible subjects $(44.8 \%, P<.001)$ (see the supplementary materials at http://www.rcjournal.com). Nonetheless, after excluding moribund subjects from the analysis, the mortality risk between RCT-eligible subjects and RCT-ineligible subjects remained significant (hazard ratio 0.57 [95\% CI 0.49-0.67], $P<.001)$. In addition, there was no pattern suggesting a consistent reduction in mortality over the $16-\mathrm{y}$ study period (see the supplementary materials at http:// www.rcjournal.com).

Regardless of RCT eligibility or ineligibility, achieving a $\mathrm{P}_{\text {plat }} \leq 30 \mathrm{~cm} \mathrm{H}_{2} \mathrm{O}$ was associated with lower mortality (Fig. 2). However, even when stratified by $\mathrm{P}_{\text {plat }}$, RCT eligibility remained strongly associated with 90-d mortality. Overall, mortality risk was lower in subjects managed with protocol-driven versus clinician-driven lung-protective ventilation (hazard ratio 0.60 [95\% CI 0.52-0.69], $P<.001$ ), even among subjects whose $\mathrm{P}_{\text {plat }}$ was $\leq 30 \mathrm{~cm} \mathrm{H}_{2} \mathrm{O}$ (hazard ratio 0.64 [95\% CI 0.54-0.76], $P<.001)$. Among RCTineligible subjects, mortality also was significantly lower among protocol-managed subjects with $\mathrm{P}_{\text {plat }} \leq 30 \mathrm{~cm} \mathrm{H}_{2} \mathrm{O}$ versus clinician-driven management with a $\mathrm{P}_{\text {plat }} \leq 30 \mathrm{~cm}$ $\mathrm{H}_{2} \mathrm{O}$ (hazard ratio 0.67 [95\% CI 0.54-0.83], $P<.001$ ).

Factors contributing to $90-\mathrm{d}$ mortality in multivariate analysis included age, APACHE II score, RCT ineligibility, Berlin classification severity, and number of ancillary therapies (Table 3). In contrast, a $\mathrm{P}_{\text {plat }} \leq 30 \mathrm{~cm} \mathrm{H}_{2} \mathrm{O}$ and development of ARDS in either a surgical or neurocritical care setting were associated with decreased 90-d mortality.

\section{Secondary Outcomes}

Among survivors, mechanical ventilation duration, ICU LOS, and hospital LOS (following ARDS onset) were 
significantly shorter in the RCT-eligible group (Table 1). However, these differences may be attributable to the inclusion of subjects with acute brain injury, whose mechanical ventilation duration, ICU LOS, and hospital LOS were significantly longer than non-brain-injured RCT-ineligible subjects, as well as RCT-eligible subjects (see the supplementary materials at http://www.rcjournal.com). When acute brain injury subjects were removed from the analysis, there were no differences in any of these variables between other RCT-ineligible and RCT-eligible subjects.

\section{Discussion}

Our main finding was that RCT-eligible subjects managed with lung-protective ventilation had markedly lower mortality compared to RCT-ineligible subjects at day 90 despite having similar severity of acute lung injury and benefiting from similar quality of lung-protective ventilation. This remained true after excluding subjects deemed moribund because of apparent refractory shock. Consistent with other studies, our results indicate that RCT-ineligible subjects had significantly higher illness severity scores at ARDS onset. In those who survived to hospital discharge, RCT-eligible subjects had significantly fewer days of mechanical ventilation and shorter ICU LOS and hospital LOS compared to RCT-ineligible subjects. These particular findings may be explained in part by the presence of subjects with acute brain injury, who accounted for $\sim 35 \%$ of the RCT-ineligible study cohort.

Mortality was extraordinarily high among RCT-ineligible subjects in specific subsets, namely Berlin classification of severe ARDS (62\%), nonpulmonary sepsis (72\%), other less common etiologies (67\%), end-stage liver disease (73\%), and those deemed moribund (83\%). In contrast, subgroups of RCT-ineligible subjects with acute brain injury, pneumonia, and trauma had lower mortality rates of $43 \%, 37 \%$, and $36 \%$, respectively, which were similar to crude mortality rates reported in the general ARDS population. ${ }^{18-22}$ Overall mortality risk among RCT-ineligible subjects was more than twice that of RCT-eligible subjects (hazard ratio 2.26 [95\% CI 1.95-2.63], $P<.001)$.

A systematic review and meta-analysis of studies between 1994 and 2006 reported significantly lower pooled mortality among subjects enrolled into RCTs versus observational studies (36.2\% vs $44.0 \%$, respectively); observational studies were associated with a substantially higher mortality risk (odds ratio 1.36 [95\% CI 1.081.73]. ${ }^{2}$ Observational study subjects included both those who would have met RCT-eligibility criteria and those who would have been ineligible for RCTs. Moreover, approximately half of the time period covered by these studies was prior to publication of the seminal ARDSNet study $^{1}$ and more widespread adoption of lung-protective ventilation.
In contrast, our study sample spanned $\sim 16 \mathrm{y}$ at an original ARDSNet study site that quickly adopted the ventilator protocol for clinical management, ${ }^{5}$ in which the majority of subjects (58\%) were RCT-eligible. These factors likely account for our lower crude mortality rate of $37 \%$ for the entire study sample. Despite less rigid adherence to the ARDSNet protocol, mortality among our RCT-eligible subjects (with some exceptions) was similar to that reported in several ARDSNet studies, with the exception being the aerosolized albuterol trial (see the supplementary materials at http://www.rcjournal.com). ${ }^{1,8,23-26}$

The generalizability of our findings is limited because of 3 factors, some of which may be unique to our institution. First, we approach ARDS surveillance consistent with our participation as an ARDSNet clinical trials site from 1996 to 2008. This includes daily screening for early identification of at-risk patients, rapidly detecting ARDS onset, and strongly advocating use of the ARDSNet protocol. Second, our highly skilled respiratory therapists were individually trained in (and had used) the ARDSNet ventilator protocols continuously since 1996. Third, these efforts were facilitated by consistent, strong cross-disciplinary physician support for lung-protective ventilation.

Others have speculated that mortality rates between therapeutic RCTs and observational studies might be reduced by more stringent adherence to lung-protective ventilation protocols. ${ }^{3}$ Our study supports this notion. Within hours of ARDS recognition, $73 \%$ of our subjects were ventilated at a $\mathrm{V}_{\mathrm{T}} \leq 8 \mathrm{~mL} / \mathrm{kg}$ and $92 \%$ at $\mathrm{V}_{\mathrm{T}} \leq 9 \mathrm{~mL} / \mathrm{kg}$. $\mathrm{P}_{\text {plat }}$ was universally monitored, and $85 \%$ of subjects had a $\mathrm{P}_{\text {plat }} \leq 30$ $\mathrm{cm} \mathrm{H}_{2} \mathrm{O}$ within that time frame. Furthermore, $74 \%$ of subjects managed by protocol experienced additional reductions in $\mathrm{V}_{\mathrm{T}}, \mathrm{P}_{\text {plat }}$, and elastic driving pressure within $24 \mathrm{~h}$ of protocol initiation.

In contrast, a 50-nation observational study reported that $<67 \%$ of ARDS subjects were managed with a $\mathrm{V}_{\mathrm{T}} \leq 8$ $\mathrm{mL} / \mathrm{kg}$, while $\mathrm{P}_{\text {plat }}$ was monitored in $<40 \%$ with corresponding hospital mortality rates of $34.9 \%$ (95\% CI 31.4 $38.5 \%$ ) for mild ARDS, $40.3 \%$ (95\% CI 37.4-43.3\%) for moderate ARDS, and 46.1\% (95\% CI 41.9-50.4\%) for severe ARDS. ${ }^{18}$ When comparing our RCT-eligible data to multi-center RCT data used by Force et al, ${ }^{7}$ our hospital mortality was below the $95 \%$ CI for mild ARDS (19.5\% vs 24-30\%), moderate ARDS (24.4\% vs 29-34\%), and severe ARDS $(38.3 \%$ vs $42-48 \%){ }^{7}$

Our study addresses some previously cited limitations of applying RCT study results to the general ARDS population, namely the prevalence of higher nonenrollment into RCTs among public hospitals caring for vulnerable populations. ${ }^{3}$ San Francisco General Hospital provides care primarily to this patient population. Early identification and enrollment of RCT-eligible subjects is another factor that limits the ability to generalize of lung-protective ventilation RCT results to the ARDS population at 


\section{ARDS Outcomes In Non-Research SubJects}

large. ${ }^{3}$ Following announcement of the seminal ARDSNet study results in the spring of 1999, we made a concerted effort to identify patients with ARDS quickly and to encourage implementation of the ARDSNet ventilator protocol. $^{5}$

We previously reported that our formal adoption of the ARDSNet ventilator protocol (2000-2003) reduced hospital mortality compared to clinical practice (1998-1999) both in RCT-eligible (from $40 \%$ to $23 \%, P=.02$ ) and RCT-ineligible subjects (from $78 \%$ to $48 \%, P=.031$ ), which indicates that the ARDSNet protocol improved survival regardless of mortality risk categorization. ${ }^{5}$ Our current study extends these findings and suggests that even initiating less structured lung-protective ventilation soon after ARDS onset reduces mortality risk.

Our study is limited by our reliance upon data gathered for quality assurance purposes. That data had to be abstracted by hand necessitated practical limitations on the amount of data that could be collected (eg, our inability to collect Sequential Organ Failure Assessment scores, fluid balance, ventilator settings over an extended time period, sedative use). Therefore, our data lack much of the "granularity" that could provide greater control of important confounders and, therefore, a more refined interpretation of our results. Although our impression was that day-to-day ventilator management was reasonably constant, we have no data to support it.

\section{Conclusions}

Among subjects with ARDS who were identified early and managed primarily using the ARDSNet ventilator protocol, those who met RCT enrollment eligibility criteria had a mortality rate similar to that reported in several ARDSNet trials. Moreover, RCT-eligible subjects had a $>50 \%$ reduction in mortality risk compared to RCT-ineligible subjects.

\section{REFERENCES}

1. Acute Respiratory Distress Syndrome Network, Brower RG, Matthay MA, Morris A, Schoenfeld D, Thompson BT, Wheeler A. Ventilation with lower tidal volumes as compared with traditional tidal volumes for acute lung injury and the acute respiratory distress syndrome. $\mathrm{N}$ Engl J Med 2000;342(18):1301-1308.

2. Phua J, Badia JR, Adhikari NK, Friedrich JO, Fowler RA, Singh JM, et al. Has mortality from acute respiratory distress syndrome decreased over time?: A systematic review. Am J Respir Crit Care Med 2009; 179(3):220-227.

3. Pais FM, Sinha P, Liu KD, Matthay MA. Influence of clinical factors and exclusion criteria on mortality in ARDS observational studies and randomized controlled trials. Respir Care 2018;63 (8):1060-1069.

4. Morris AH, Wallace CJ, Menlove RL, Clemmer TP, Orme JF Jr, Weaver LK, et al. Randomized clinical trial of pressure-controlled inverse ratio ventilation and extracorporeal $\mathrm{CO} 2$ removal for adult respiratory distress syndrome. Am J Respir Crit Care Med 1994;149(2 Pt 1):295-305.
5. Kallet RH, Jasmer RM, Pittet JF, Tang JF, Campbell AR, Dicker R, et al. Clinical implementation of the ARDS network protocol is associated with reduced hospital mortality compared with historical controls. Crit Care Med 2005;33(5):925-929.

6. Bernard GR, Artigas A, Brigham KL, Carlet J, Falke K, Hudson L, et al. The American-European Consensus Conference on ARDS. Definitions, mechanisms, relevant outcomes, and clinical trial coordination. Am J Respir Crit Care Med 1994;149(3 Pt 1):818-824.

7. Force ADT, Ranieri VM, Rubenfeld GD, Thompson BT, Ferguson ND, Caldwell E, et al. Acute respiratory distress syndrome: the Berlin Definition. JAMA 2012;307(23):2526-2533.

8. Brower RG, Lanken PN, MacIntyre N, Matthay MA, Morris A, Ancukiewicz M, et al. Higher versus lower positive end-expiratory pressures in patients with the acute respiratory distress syndrome. $\mathrm{N}$ Engl J Med 2004;351(4):327-336.

9. Knaus WA, Draper EA, Wagner DP, Zimmerman JE. APACHE II: a severity of disease classification system. Crit Care Med 1985;13 (10):818-829.

10. Le Gall JR, Lemeshow S, Saulnier F. A new Simplified Acute Physiology Score (SAPS II) based on a European/North American multicenter study. JAMA 1993;270(24):2957-2963.

11. Murray JF, Matthay MA, Luce JM, Flick MR. An expanded definition of the adult respiratory distress syndrome. Am Rev Respir Dis 1988;138(3):720-723.

12. Amato MB, Meade MO, Slutsky AS, Brochard L, Costa EL, Schoenfeld DA, et al. Driving pressure and survival in the acute respiratory distress syndrome. N Engl J Med 2015;372(8):747-755.

13. Ortiz RM, Cilley RE, Bartlett RH. Extracorporeal membrane oxygenation in pediatric respiratory failure. Pediatr Clin North Am 1987;34 (1):39-46.

14. Sinha P, Calfee CS, Beitler JR, Soni N, Ho K, Matthay MA, Kallett RH. Physiologic analysis and clinical performance of the ventilatory ratio in acute respiratory distress syndrome. Am J Respir Crit Care Med 2019;199(3):333-341.

15. Peek GJ, Mugford M, Tiruvoipati R, Wilson A, Allen E, Thalanany $\mathrm{MM}$, et al. Efficacy and economic assessment of conventional ventilatory support versus extracorporeal membrane oxygenation for severe adult respiratory failure (CESAR): a multicentre randomised controlled trial. Lancet 2009;374(9698):1351-1363.

16. Slutsky AS. Consensus conference on mechanical ventilation-January 28-30, 1993 at Northbrook, Illinois, USA. Part 2. Intensive Care Med 1994;20(2):150-162.

17. Hubmayr RD, Kallet RH. Understanding pulmonary stress-strain relationships in severe ARDS and its implications for designing a safer approach to setting the ventilator. Respir Care 2018;63 (2):219-226.

18. Bellani G, Laffey JG, Pham T, Fan E, Brochard L, Esteban A, et al. Epidemiology, patterns of care, and mortality for patients with acute respiratory distress syndrome in intensive care units in 50 countries. JAMA 2016;315(8):788-800.

19. Villar J, Blanco J, Añón JM, Santos-Bouza A, Blanch L, Ambrós A, et al. The ALIEN study: incidence and outcome of acute respiratory distress syndrome in the era of lung protective ventilation. Intensive Care Med 2011;37(12):1932-1941.

20. Cooke CR, Kahn JM, Caldwell E, Okamoto VN, Heckbert SR, Hudson LD, et al. Predictors of hospital mortality in a populationbased cohort of patients with acute lung injury. Crit Care Med 2008;36 (5): 1412-1420.

21. Máca J, Jor O, Holub M, Sklienka P, Burşa F, Burda M, et al. Past and present ARDS mortality rates: a systematic review. Respir Care 2017;62(1):113-122.

22. Zambon M, Vincent JL. Mortality rates for patients with acute lung injury/ARDS have decreased over time. Chest 2008;133(5):1120-1127. 


\section{ARDS OUtCOMES IN Non-ResEARCh SubJeCtS}

23. National Heart, Lung, and Blood Institute ARDS Clinical Trials Network, Wiedemann HP, Wheeler AP, Bernard GR, Thompson BT, Hayden D, et al. Comparison of two fluid-management strategies in acute lung injury. N Engl J Med 2006;354(24):25642575.

24. Zhang Z, Spieth PM, Chiumello D, Goyal H, Torres A, Laffey JG, Hong Y. Declining mortality in patients with acute respiratory distress syndrome: an analysis of the ARDS Network trials. Crit Care Med 2019;47(3):315-323.
25. National Heart, Lung, and Blood Institute PETAL Clinical Trials Network, Moss M, Huang DT, Brower RG, Ferguson ND, Ginde AA, et al. Early neuromuscular blockade in the acute respiratory distress syndrome. N Engl J Med 2019;380(21):1997-2008.

26. National Heart, Lung, and Blood Institute ARDS Clinical Trials Network, Matthay MA, Brower RG, Carson S, Douglas IS, Eisner M, et al. Randomized, placebo-controlled clinical trial of an aerosolized beta(2)-agonist for treatment of acute lung injury. Am J Respir Crit Care Med 2011;184(5):561-568.

This article is approved for Continuing Respiratory Care Education credit. For information and to obtain your CRCE

(free to AARC members) visit

www.rcjournal.com 